\title{
Sperm storage reflects within- and extra-pair mating opportunities in a cooperatively breeding bird
}

Sjouke A. Kingma ${ }^{1,2} *$, Michelle L. Hall ${ }^{1,3}$, and Anne Peters ${ }^{1,4}$

5

${ }^{1}$ Max Planck Institute for Ornithology, Schlossallee 2, 78315, Radolfzell, Germany

${ }^{2}$ School of Biological Sciences, University of East Anglia, Norwich, NR4 7TJ, UK

${ }^{3}$ Department of Zoology, University of Melbourne, Melbourne, Victoria, 3010, Australia

${ }^{4}$ School of Biological Sciences, Monash University, Clayton, Victoria, 3800, Australia

10 S.kingma@uea.ac.uk $(*$ corresponding author)

hall.m@unimelb.edu.au

Anne.Peters@monash.edu

15 Short title: temporal variation in cloacal protuberance size

Keywords: cloaca, intra-sexual competition, sperm competition, post-copulatory sexual selection, extra-pair paternity, Malurus 


\begin{abstract}
In passerine birds, storage and maturation of sperm takes place in the cloacal protuberance (CP),

25 an external swelling of the reproductive organ. The considerable variation in $\mathrm{CP}$ size among species is presumed to be a consequence of varying levels of sperm-competition, but whether individual variation in $\mathrm{CP}$ size within a species also reflects sperm competition is not well established. Here, we study temporal variation in male CP size in relation to within-pair and extra-pair mating opportunities and cuckoldry risk in purple-crowned fairy-wrens Malurus

30 coronatus. This is a socially monogamous cooperatively breeding passerine that can breed yearround and has low levels of extra-pair paternity (in $6 \%$ of broods). We show that male CP size sharply increased a few weeks before, and rapidly regressed after his partner laid eggs, consistent with a cost of its maintenance and/or sperm production. Surprisingly, despite low levels of extrapair paternity, CP size of non-breeding and pre-breeding males was positively correlated with the

35 number of breeding females in the population, suggesting that $\mathrm{CP}$ size is sensitive to extra-pair mating opportunities. However, CP sizes do not seem to reflect cuckoldry risk: CP size of dominant males was unaffected by the presence of a subordinate that was unrelated to the dominant female, although those subordinates occasionally sire offspring, and had a larger CP than subordinates living with their mother. Our results suggest that, even in a species with low

40 levels of extra-pair paternity, individual investment in sperm storage reflects both within-pair and, albeit to lesser extent, extra-pair mating opportunities.
\end{abstract}




\section{INTRODUCTION}

50 Much of the variation in male reproductive anatomy is believed to be the evolutionary result of differences in sperm competition (Birkhead et al. 1991, 1993; Briskie 1993; Tuttle et al. 1996). When more than one male is competing to fertilise the eggs of a female, sperm competition will lead to the evolution of traits that increase a male's chances of achieving fertilization. Since sperm production is limited and costly, males are expected to invest strategically in such traits,

55 according to female promiscuity and additional mating opportunities (Wedell et al. 2002; Pizzari et al. 2003). Female promiscuity in the form of multiple mating is common in birds, even those that are socially monogamous (Griffith et al. 2002), resulting in adaptations to sperm competition (Birkhead and Møller 1998). One adaptation is the cloacal protuberance (CP), an external swelling of the reproductive organs that develops when males breed. The $\mathrm{CP}$ forms during

60 seasonal testicular hypertrophy and sperm production, and functions in storage and maturation of sperm (Wolfson 1952). The CP thus enables males to store large numbers of spermatozoa. When sperm competition is high, larger CPs can improve fertilization success by maintaining sufficient stored sperm for frequent copulations or for large sperm transfers (Salt 1954; Wolfson 1954; Lake 1981; Birkhead et al. 1991, 1993). Since maintenance of tissue and sperm production is costly

65 (Dewsbury 1982; Nakatsuru and Kramer 1982; Pitnick and Markow 1994; Olsson et al. 1997), CP size is likely optimised strategically, according to cost-benefit trade-offs. This should not only apply to seasonal enlargement and regression of CPs at the level of the population, but also to individual variation in $\mathrm{CP}$ size among males.

Most research has focused on CP size differences among species, showing that species

70 with large CPs often have a polyandrous mating system or mate promiscuously (e.g., Briskie 1993, Tuttle et al. 1996). Because temporal variation in CP size has only been studied in species with rather high levels of sperm competition and/or unusually large CP (e.g., Nakamura 1990; Briskie 1993; Mulder and Cockburn 1993; Sax and Hoi 1998), it is unclear whether and how individual and temporal variation in $\mathrm{CP}$ size reflects levels of sperm competition more broadly. 
be expected that males in socially monogamous species with high levels of EP paternity maintain large CPs during the entire breeding season to enable successful EP fertilizations (e.g., Low et al. 2005). In superb fairy-wrens Malurus cyaneus, a species with intense sperm competition (61-76\% EP offspring; Mulder et al. 1994; Double and Cockburn 2003), CPs remain large throughout the

80 breeding season at the population level, indicating that extra-pair rather than within-pair mating opportunities explain temporal variation in CP size (Nakamura 1990). In male tree-swallows Tachycineta bicolor, however, male CPs regress soon after breeding of their social mate (Lombardo 2001). Although a high proportion of offspring in this species is EP (69\%; Barber et al. 1996), this regression is likely the result of a relatively short and synchronous breeding season,

85 leading to reduced EP mating opportunities when a male's own reproductive attempt is nearly completed (Lombardo 2001). In bearded tits Panurus biarmicus, male CP size also tracks withinpair mating opportunities, as CPs regress between breeding attempts (although they remain larger than during non-breeding; Sax and Hoi 1998). As these birds have moderate levels of EP paternity (14\% EP offspring; Hoi and Hoi-Leitner 1997), the difference between this species and

90 M. cyaneus suggests that temporal variation in CP size reflects individual variation in degree of sperm competition experienced. However, individual variation in CP size has, as far as we are aware, not been studied in detail in a species with low levels of sperm competition, which will be useful for making inferences about the relative importance of within-pair breeding and sperm competition in explaining temporal changes in $\mathrm{CP}$ size.

95 In this study, we investigate how individual CP size varies with the stage of the withinpair breeding cycle, extra-pair mating opportunities and cuckoldry risk in male purple-crowned fairy-wrens $M$. coronatus. These socially monogamous birds breed cooperatively, with a dominant breeding pair assisted by subordinate helpers (Rowley and Russell 1993, 1997; Kingma et al. 2011a). Dominant females can initiate breeding attempts year-round and most offspring are 100 sired by the social father (the dominant male), with only $7 \%$ of offspring sired by other males 
(Kingma et al. submitted ms). Consequently, we predict that $\mathrm{CP}$ size of male $M$. coronatus closely synchronises with the within-pair female's reproductive cycle, rather than with extra-pair mating opportunities. We test this prediction by relating dominant male $\mathrm{CP}$ size to breeding stage and days to egg-laying of his social mate, as well as the number of EP mating opportunities in the

105 population. Additionally, since male subordinates that are unrelated to the dominant female can gain within-group paternity (Kingma et al. 2009), we quantify whether CP is affected by such a situation of cuckoldry risk (for dominant males), and within-group mating opportunity (for subordinate males).

\section{METHODS}

We studied a colour-banded population of M. coronatus resident along Annie Creek and the Adcock River in Mornington Wildlife Sanctuary in Western Australia ( ${\mathrm{S} 17^{\circ}}^{3} 1^{\prime} \mathrm{E} 126^{\circ} 6^{\prime}$ ), from 2005 to 2010 (Hall and Peters 2008, 2009; Kingma et al. 2010, 2011b). The species is restricted to riparian vegetation and groups maintain all-purpose territories year-round that are linearly

115 arranged along creeks and rivers. M. coronatus is a cooperatively breeding species in which the socially monogamous pair may be accompanied by a number of male and female subordinates (Kingma et al. 2011a, b). In our population, birds can breed in any month of the year, with typically a major peak in breeding activity during the wet season (Dec-Mar), and a minor peak in breeding activity in the late dry season in some years (Sep-Oct; Rowley and Russell 1997;

120 unpubl. data). During year-round weekly population censuses, we documented group size, and social status (dominant or subordinate) of each uniquely colour-banded group member based on behavioural cues (the most obvious that only the breeding pair sing duets for cooperative territorial defence; Hall and Peters 2008). Only dominant females build the nest (Rowley and Russell 1993, 1997) and during each census we searched for nests by following the dominant

125 female for at least 20 minutes. Nests were checked regularly to determine laying date and number of eggs (modal clutch size is 3 eggs) and nestlings. We obtained a very accurate estimate of 
breeding progress for the population throughout the year: we rarely missed a reproductive attempt, as evidenced by the fact that $>88 \%$ of nests $(N=629)$ are found during nest building and incubation, and $<3 \%$ of nests are found by the appearance of fledglings (unpubl. data).

130 Throughout the study period birds were caught using mist nets, and we measured CP size (see below) and tarsus length $( \pm 0.1 \mathrm{~mm}$ using a sliding caliper $)$.

Paternity analysis showed that extra-pair paternity is rare in M. coronatus: of 509 offspring (217 broods) between 2006 and 2010, genotyping showed that 29 (5.7\%) offspring from $15(6.9 \%)$ broods were extra-pair. Of these, 25 (4.9\%) offspring from $13(6.0 \%)$ broods were

135 sired by an extra-group (EG) dominant male (Kingma et al. submitted ms; see also Kingma et al. 2009 with a smaller dataset). Subordinates are often offspring from the breeding pair and subordinates that are related to the female do not attain within-group paternity. However, due to death or dispersal, subordinates can be unrelated to the dominant female (Kingma et al. 2011a). These helpers do occasionally sire within-group offspring: the same analysis (217 broods, 509

140 offspring) showed that 4 offspring from 2 broods (1\%) were sired by a subordinate male within the same group that was not related to the dominant female (Kingma et al. 2011a) and 7\% (of 30) such helpers gained within-group paternity. Unrelated helpers thus form a reproductive threat to the dominant male that is of similar magnitude as the risk of cuckoldry by extra-group males.

\section{CP measurements}

The cylinder-shaped CP of males was initially measured as width (w) and length (l) (perpendicular to each other; lateral and antero-posterior axes respectively), using a sliding caliper to the nearest $0.1 \mathrm{~mm}$. The 'area' of the CP was calculated using the formula $(\mathrm{w} / 2+1 / 2)^{2} *$ $\pi$. Later in the study, we also measured height (h), and calculated CP volume following the

150 formula: $(\mathrm{w} / 2+1 / 2)^{2} * \pi * \mathrm{~h}$ (Briskie 1993). Because area and volume calculations were highly correlated $\left(r^{2}=0.901, N=158\right.$ birds, volume $=3.897 *$ area -8.133$)$, we decided to use 'area' to allow inclusion of all samples, and we refer to this as ' $\mathrm{CP}$ size'. 


\section{Statistical analyses}

155 We related CP size to opportunity for fertilizations and reproductive competition. For dominant males, we examined the relationship with within-pair breeding stage and number of days from the start of egg-laying of their (within-pair) female as well as EP mating opportunities (the number of actively breeding females in the population). We quantified the effect of within-group reproductive competition (a subordinate unrelated to the dominant female) on dominants and 160 subordinates.

For the first set of analyses, those involving within-pair breeding stage, number of days from the start of egg-laying and EP mating opportunities (see below), we used a mixed modelling approach using the nlme-package in R 2.11.1 (Pinheiro et al. 2011, R development core team 2011), including bird-identity as random variable because some males were included in the

165 analyses more than once. We included 'CP size' as the response variable, and we excluded nonsignificant $(P>0.05)$ independent variables (see below) using backwards elimination. Reported values are obtained after re-inclusion in the model with only statistically significant variables. Effect sizes $(\beta)$ are reported as average \pm SE. Normal distribution and homogeneity of variance of the model residuals were confirmed by visual inspection. CP size was log transformed in all

170 analyses, to normalise model residuals (except for analyses with pre-breeding dominant males only; see below). We included tarsus length in the models to correct for structural size, but this was unrelated to $\mathrm{CP}$ size in all analyses (all: $P>0.14$ ). Additionally, we included age (days since hatching) in the models using a subset of dominant males with known ages (in 99 of 144 cases we did not know exact age as these males were immigrants or already present in the beginning of the 175 study). In all analyses the effect of age on CP size was non-significant (all: $P>0.13$ ). 


\section{Within-pair breeding stage}

To analyze whether $\mathrm{CP}$ size varied with within-pair breeding stage, for each dominant male we

180 classified the breeding stage on his home territory as non-breeding, pre-breeding, incubating, or with dependent young at time of capture. Because breeding can take place throughout the year, we based this on the nesting attempt that was closest in time to capture of the male. We classified birds as non-breeding when they were more than 3 weeks before egg-laying (average $\pm \mathrm{SE}=106$ \pm 7.3 days, range $=26$ to 274 days, $N=59$ ), after a failed nesting attempt or 6 weeks after

185 fledging (average $\pm \mathrm{SE}=135 \pm 11.9$ days after egg-laying, range $=53$ to 250 days, $N=20$ ). Prebreeding was defined as between 3 weeks and 1 day before egg-laying (average \pm SE $=6 \pm 0.9$ days, range $=1$ to 20 days, $N=33$ ); this also included nest-building for nests in which no eggs were laid $(N=9)$. Incubation included all males that had a nest with eggs (average $\pm \mathrm{SE}=8 \pm 1.5$ days after start of egg-laying, range $=0$ to $14, N=9$ ); we included here two cases where the

190 female was egg-laying since close proximity between partners (physical mate guarding) ceases when the first egg is laid and within-pair copulations stop occurring 2 days before egg-laying (Hall and Peters 2009). Finally, we defined dependent young as nestlings (average $\pm \mathrm{SE}=25 \pm$ 0.6 days after egg-laying, range $=22$ to 26 days, $N=6$ ), and dependent fledglings (less than 6 weeks after fledging; average $\pm \mathrm{SE}=54 \pm 3.0$ days after egg-laying, range $=45$ to 67 days, $N=$

195 7). We excluded one male (with nestlings) from this analysis as this male was identified as an outlier (using Grubb's test for outliers; Barnett and Lewis 1994) because of an exceptionally large CP $\left(27.1 \mathrm{~mm}^{2}\right)$. In a separate model, we analyzed whether the number of days from the start of egg-laying predicted $\mathrm{CP}$ size. Because the range of number of days from the start of egg-laying was large (see above, and Fig. 2), we $\log (+1)$-transformed number of days from the start of egg-

200 laying. Two non-breeding males did not have a breeding attempt during their tenure as dominant males, so we could not calculate the number of days from egg-laying for these males.

Population breeding activity - extra-pair mating opportunities 
We assessed whether EP-mating opportunities predict male $\mathrm{CP}$ size by relating $\mathrm{CP}$ size to the 205 number of females actively breeding in the population. To control for confounding effects of CP enlargement due to their own breeding, we performed two analyses: one including only nonbreeding males and one including only pre-breeding males. Additionally, to control for potential co-variation between breeding activity in the population and the likelihood that his own mate was about to initiate a breeding attempt, we included number of days from the start of egg-laying for

210 each focal male in both analyses (absolute or $\log (+1)$ transformed; excluding the two males that did not have a clutch (see above)). For each male, we calculated the number of clutches in the population initiated in the month of capture of the focal male (as proxy for EP-mating opportunities; not including within-pair clutches). Because the timing of breeding was slightly different between birds on Annie Creek and the Adcock River (that have different flow regimes;

215 Hall, Kingma and Peters, personal observation), we calculated the number of clutches initiated for both rivers separately. The total number of territories changed slightly over the course of the study and differed between rivers, so we also calculated the proportion of territories that initiated a clutch in each month for each river. Using the latter approach, results were similar (and therefore not reported).

\section{Within-group reproductive competition}

To make inferences about $\mathrm{CP}$ size and within-group reproductive competition, we included only males in the pre-breeding stage, as direct reproductive competition takes place during this period. We compared groups with related subordinates (living with their mother), and unrelated

225 subordinates (not living with a first or second order relative) that might gain paternity in the group (Kingma et al. 2009, 2011a). CP sizes of subordinate males that were either living with their mother or unrelated to the dominant female were compared using a linear model (we did not use a mixed model as none of the 21 subordinates was included twice). We also included subordinate age in the model (number of days, as independent variable). Additionally, to assess 
230 the effect of cuckoldry risk on dominant males, we compared dominant males living in a group with a subordinate male unrelated to the dominant female to those that did not have subordinates unrelated to the female (in a mixed model, as above, and correcting for the number of clutches in the population initiated in the month of capture of the focal male). For a subset of males for which number of days until egg-laying was known (excluding 9 males that were captured when

235 their female was nest-building but did not lay eggs in that nest), we repeated this model controlling for number of days until egg-laying.

\section{RESULTS}

\section{Within-pair breeding stage}

240 CP size of dominant males varied considerably over the course of the breeding cycle on their own territory (Fig. 1). CPs of non-breeding males were significantly smaller than CPs of males with or approaching an active breeding attempt (difference between non-breeding males and pre-breeding males $(\beta=0.308 \pm 0.0318, t=9.461, P<0.001)$, and males with eggs $(\beta=0.262 \pm 0.0588, t=$ 4.366, $P<0.001)$ ), but $\mathrm{CP}$ size of males in nestling and fledgling stage was not significantly

245 different from CP size of non-breeding males $(\beta=0.0703 \pm 0.0518, t=1.358, P=0.178)$.

Number of days from the start of egg-laying was significantly correlated with CP size ( $\beta$ $=-0.230 \pm 0.0244, N=133, t=-9.450, P<0.001)$, because CPs were larger when a male's partner was closer to egg-laying (Fig. 2A).

\section{Population breeding activity - extra-pair mating opportunities}

$\mathrm{CP}$ size of non-breeding males increased with increasing numbers of females actively breeding in the population (effect of number of clutches initiated in the month of capture of the focal male: $\beta$ $=0.0277 \pm 0.00925, N=81, t=2.996, P=0.005$; Fig. 3A), but was unrelated to the number of days to egg-laying on their own territory $(\beta=-0.0000054 \pm 0.000372, N=79, t=-0.014, P=$

$2550.989 ; \log (+1)$ transformed, $\beta=-0.0405 \pm 0.0889, N=79, t=-0.455, P=0.652))$. The effects of 
population-wide breeding activity and breeding on their own territory were not confounded: there was no relationship between the number of days to egg-laying (response variable) and the number of clutches initiated in the month of capture (independent variable; $\beta=-2.316 \pm 2.896, N=79, t=$ $-0.800, P=0.430)$.

Males in the pre-breeding stage also had significantly larger CPs when more clutches were initiated in the month of capture of the focal male $(\beta=0.216 \pm 0.0919, N=42, t=2.354, P$ $=0.036$; Fig. 3B). The significant relationship between focal male CP size and the number of active breeding attempts in the month of capture remained $(\beta=0.268 \pm 0.101, N=42, t=2.649$, $P=0.033$ ) when correcting for the significant effect of number of days to egg-laying on the own

265 territory $(\beta=-0.431 \pm 0.144, t=-2.988, P=0.020$; Fig. $2 \mathrm{~B})$, using the subset of males for which this was known (see methods, $N=33$ ).

\section{Within-group reproductive competition}

In the pre-breeding stage, subordinate males that were unrelated to the dominant female had CPs

270 that were on average nearly twice as large (average $\pm \mathrm{SE}=11.88 \pm 2.06 \mathrm{~mm}^{2}, N=6$ ) as CPs of 15 subordinate males that lived with their mother (average $\pm \mathrm{SE}=6.90 \pm 0.62 ; t=-2.829, P=$ 0.011; Fig 4A). This difference was, however, non-significant $(\beta=-0.0290 \pm 0.109, t=-0.267, P$ $=0.793)$ when we statistically corrected for the highly significant effect of subordinate age $(\beta=$ $0.000548 \pm 0.000134, t=4.080, P<0.001 ;$ Fig 4A). Unrelated subordinates were older (average $275 \pm \mathrm{SE}=766 \pm 76.9$ days old; range $=542-1020)$ than related subordinates $(375 \pm 39.2$ days old; range $=167-641 ; t=4.523, P=0.002 ;$ Fig 4 A).

$\mathrm{CP}$ size of (pre-breeding) dominant males that lived in a group with a subordinate male that was unrelated to the dominant female was not statistically different from dominants that did not have any subordinate male unrelated to the dominant female $(t=-0.644, P=0.533$; Fig. 4B).

280 This result was similar $(t=-1.463, P=0.158)$ when correcting for the significant effect of 
number of days to egg-laying on the own territory (using a subset of males for which number of days to egg-laying was known exactly, see above).

\section{DISCUSSION}

\section{Within-pair breeding stage}

CP size in dominant $M$. coronatus males closely tracks the breeding cycle of their social partner.

During the 3 weeks before egg-laying, the cloacal swelling increases rapidly as egg-laying approaches (Figs. $1 \& 2$ ), and male $\mathrm{CP}$ increases in this period to, on average, nearly twice as large as that of non-breeding males (Fig. 1A). This CP enlargement is usually brief: by the

290 nestling/fledgling stage, CPs have regressed to the size of non-breeding males (Fig. 1A). Moreover, CP size of non-breeding males is not correlated with the number of days until egglaying. This transient and brief increase is in agreement with expectations of strategic allocation to sperm storage: since the maintenance of tissue and sperm production is costly (Pitnick and Markow 1994; Olsson et al. 1997), CPs should be small when the benefits of continued

295 maintenance of storing mature sperm are low. Since female $M$. coronatus can initiate breeding at any month of the year, a strategy of closely tracking his mate's breeding activities ensures male fertility at the time of within-pair copulations without redundant investment at other times. Accordingly, the decline after egg-laying, to non-breeding sized CP, appears sharper than in other species, that maintain a part- (P. biarmicus; Sax and Hoi 1998) or even full-sized (M. cyaneus;

300 Mulder and Cockburn 1993) CP outside the fertile period of their mate. This is probably related to seasonal breeding with more predictable probability of re-nesting. Additionally, or alternatively, extended maintenance of large CPs could be related to EP mating opportunities, as these species have (much) higher levels of EP paternity (14\% and 61-76\% of offspring respectively; see Introduction) than $M$. coronatus (5.7 \% EP offspring; Kingma et al.submitted 305 ms; see also Kingma et al. 2009). 


\section{Extra-pair mating opportunities}

Testes and $\mathrm{CP}$ in M. coronatus are small in comparison to their congeners that have some of the highest rates of EP mating among birds (Kingma et al. 2009). In three fairy-wrens with EP 310 paternity $>50 \%$ of broods (M. splendens, M. cyaneus and M. melanocephalus) average CP volume in breeding males is 107,105 and $123 \mathrm{~mm}^{3}$ respectively (Rowe et al. 2008; see also Kingma et al. 2009). During the pre-breeding stage, CP volume of M. coronatus males is around half as large $\left(58.2 \mathrm{~mm}^{3}, \mathrm{n}=42\right.$; all calculated as $(\mathrm{w} / 2+1 / 2)^{2} * \pi * \mathrm{~h}$ (Briskie 1993); see methods for details). We argued that this relatively small CP is the result of low competition with other

315 males over fertilizations in M. coronatus (Kingma et al. 2009).

Surprisingly however, using detailed information on breeding activity of all females in the population, we show here that when more females in the population were breeding, male $M$. coronatus grew relatively larger CPs: this occurred independent of (Fig. 3A) and in addition to (Fig. 3B) the increase as a result of within-pair breeding. This effect was larger in non-breeding

320 males compared to males with an actively breeding female (compare Fig. 3A and 3B and effect sizes), but not as large as that of within-pair breeding (compare Fig. 3A with Fig. 1: the maximum $\mathrm{CP}$ size in non-breeding males is well below the average CP size of breeding males). This increase in $\mathrm{CP}$ size in response to general breeding activity is likely related to the increasing number of EP mating opportunities, rather than an effect of males 'expecting' a within-pair

325 brood: first, we statistically controlled for the number of days to egg-laying; second, the number of days to egg-laying for a focal male and general breeding activity in the population were not related; and third, in the non-breeding stage, CP size was unrelated to the time until egg-laying. In conclusion, it seems that besides the strong correlation with within-pair breeding stage, a part of the individual variation in $\mathrm{CP}$ size consistently reflects opportunities for EP copulations in $M$. 330 coronatus, despite low EP paternity in this species.

Although we have never observed EP copulations in M. coronatus (Kingma et al. 2009), in $M$. cyaneus, fertile females initiate clandestine pre-dawn forays for extra-pair copulations 
(Double and Cockburn 2000) and males' reproductive state (individual testosterone level) tracks the number of fertile females in the population (Peters et al. 2001). If males similarly track extra-

335 pair opportunities in M. coronatus, our results suggest that males maintain an elevated level of 'reproductive-readiness' when the likelihood that EP females visit them for copulations increases. In agreement with this suggestion, in M. melanocephalus (attractive) males that have more EP mating opportunities have larger CPs than unattractive males (Karubian 2002; Rowe et al. 2010).

\section{Within-group reproductive competition: subordinate vs. dominant?}

Subordinate male M. coronatus had smaller CPs than dominant males (Fig. 4), which has also been found in other cooperative breeders where subordinates have limited mating opportunities (e.g. Brouwer et al. 2009). Interestingly, in the closely related M. cyaneus and M. splendens subordinate males have similarly sized CPs as dominant males (Mulder and Cockburn 1993,

345 Rowe and Pruett-Jones 2006; but see also Rowe et al. 2010). In these species, levels of EP paternity are extremely high, and although subordinates are less successful than dominants, they can still gain fairly substantial reproductive success (Cockburn et al. 2003, 2009, Webster et al. 2004). Similarly, we show here that subordinate male $M$. coronatus that live in a group with an unrelated dominant female had a larger CP than those living with their mother, approaching in

350 size the $\mathrm{CP}$ of breeding dominant males (Fig. 4A). This may simply be due to unrelated subordinates being older. However, it also reflects increased mating opportunity since subordinates with an unrelated female occasionally sire offspring (in $7 \%$ of 30 cases), whereas subordinates that live with their mother never sire offspring (0\% of 92 cases; Kingma 2011a). Greater reproductive readiness in unrelated subordinate males has been demonstrated in other

355 cooperative breeders. For example, in the classic example of the pied kingfisher Ceryle rudis, 'secondary helpers' (immigrants) are unrelated to the dominant female and have testosterone levels similar to those of dominant males, whereas 'primary helpers' (offspring of the breeding 
pair) express low levels of testosterone, have small gonads and do not produce sperm (Reyer et al. 1986), and they presumably also had smaller CPs.

360 Dominant males did not show any signs of defensively investing more in sperm competition when the risk of cuckoldry increased: when there was a subordinate in the group that was unrelated to the dominant female (that occasionally gain within-group paternity) the dominant male did not have a larger CP. Possibly males are relying more on behavioural paternity assurance, since males maintain very close proximity to their partners before laying (Hall and

365 Peters 2009). Behavioural strategies to minimise sperm competition are common, and can be effective (Komdeur et al. 1999; Chuang-Dobbs et al. 2001; Brylawski and Whittingham 2004). For example, in $C$. rudis, dominant males do not tolerate secondary helpers during the copulatory phase, although no paternity analysis was performed to quantify cuckoldry (Reyer et al. 1986). Although we occasionally observed displacement of unrelated subordinates by dominant males in

370 M. coronatus (Hall and Peters 2009), dominant male behavioural strategies are not $100 \%$ effective, as $7 \%$ of subordinates that are unrelated to the dominant female do gain within-group paternity (Kingma et al. 2009, 2011a). Possibly, since the unrelated (to the female) subordinate is not only a reproductive competitor, but often also a son of the dominant male (Kingma et al. 2009), there might be reduced fitness incentive for investment in sperm competition. 375 Alternatively, our results could suggest that defensive sperm competition strategies require different investment compared to offensive sperm competition strategies, as has been shown for sperm morphology in M. cyaneus (Calhim et al. 2011).

Altogether, our data suggest that, in a species with limited extra-pair paternity, individual 380 variation in sperm storage of dominant and subordinate males reflects primarily the within-pair breeding stage, and to a lesser extent extra-pair mating opportunities, as predicted by strategic investment in sperm competition. Further studies incorporating EP mating opportunities in 
explaining individual variation in $\mathrm{CP}$ size will need to confirm whether this is a general pattern among species, including those with low levels of EP paternity.

\section{ACKNOWLEDGMENTS}

We are grateful to the staff of Mornington Wildlife Sanctuary and the Australian Wildlife Conservancy for their superb logistical support, and our excellent team of field assistants. We thank Kaspar Delhey for comments on the manuscript. This research was funded by the Minerva

390 Program for Women in Science of the Max Planck Society (to A.P.), and by the Netherlands Organisation for Scientific Research (NWO; to S.A.K.).

\section{ETHICAL STANDARDS}

All fieldwork was performed with permission from the Max Planck Institute for Ornithology

395 Animal Ethics Committee, the Australian Wildlife Conservancy, the Western Australia Department of Conservation and Land Management (licenses BB002178 and BB002311), and the Australian Bird and Bat Banding Scheme (Authority 2230 and 2073).

The authors declare that they have no conflict of interest.

\section{REFERENCES}

Barber CA, Robertson RJ, Boag PT (1996) The high frequency of extra-pair paternity in Tree Swallows is not an artifact of nest-boxes. Behav Ecol Sociobiol, 38:425-430.

Barnett V, Lewis T (1994) Outliers in statistical data, 3rd edn. John Wiley and Sons, New York

Birkhead TR, Briskie JV, Møller AP (1993) Male sperm reserve and copulation frequency in birds. Behav Ecol Sociobiol 32:85-93.

Birkhead TR, Hatchwell BJ, Davies NB (1991) Sperm competition and the reproductive organs of the male and the female Dunnock Prunella modularis. Ibis 133:306-311. 
Birkhead TR, Møller AP (eds) (1998) Sperm competition and sexual selection. Academic Press, London.

410 Briskie JV (1993) Anatomical adaptations to sperm competition in Smith's Longspurs and other polygynandrous passerines. Auk 110:875-888.

Brouwer L, Groothuis TGG, Vedder O, Eikenaar C, Richardson DS, Komdeur J (2009) Do primary males physiologically suppress subordinate males? An experiment in a cooperatively breeding passerine. Ethology 115:576-587.

415 Brylawski AMZ, Whittingham LA (2004) An experimental study of mate guarding and paternity in house wrens. Anim Behav 68:1417-1424.

Calhim S, Double MC, Margraf N, Birkhead TR, Cockburn A (2011) Maintenance of sperm variation in a highly promiscuous wild bird. Plos one 6:e28809.

Chuang-Dobbs HC, Webster MS, Holmes RT (2001) The effectiveness of mate guarding by male black-throated blue warblers. Behav Ecol 12:541-546.

Cockburn A, Dalziell AH, Blackmore CJ, Double MC, Kokko H, Osmond HL, Beck NR, Head ML, Wells K (2009) Superb fairy-wren males aggregate into hidden leks to solicit extragroup fertilizations before dawn. Behav Ecol 20:501-510.

Cockburn A, Osmond H, Mulder RA, Green DJ, Double MC (2003) Divorce, dispersal and incest 425 avoidance in the cooperatively breeding superb fairy-wren Malurus cyaneus. J Anim Ecol 72:189-202.

Dewsbury DA (1982) Ejaculate cost and male choice. Am Nat 119:601-610.

Double MC, Cockburn A (2000) Pre-dawn infidelity: females control extra-pair mating in superb fairy-wrens. Proc R Soc Lond B 267:465-470.

430 Double MC, Cockburn A (2003) Subordinate superb fairy-wrens (Malurus cyaneus) parasitize the reproductive success of attractive dominant males. Proc R Soc Lond B 270:379-384.

Griffith SC, Owens IPF, Thuman KA (2002) Extra pair paternity in birds: a review of interspecific variation and adaptive function. Mol Ecol 11:2195-2212. 
Hall ML, Peters A (2008) Coordination between the sexes for territorial defence in a duetting fairy-wren. Anim Behav 76:65-73.

Hall ML, Peters A (2009) Do male paternity guards ensure female fidelity in a duetting fairywren? Behav Ecol 20:222-228.

Hoi H, Hoi-Leitner M (1997) An alternative route to coloniality in the Bearded Tit: Females pursue extra-pair fertilizations. Behav Ecol 8:113-119.

440 Karubian J (2002). Costs and benefits of variable breeding plumage in the red-backed fairy-wren. Evolution 56:1673-1682.

Kingma SA, Hall ML, Segelbacher G, Peters A (2009) Radical loss of an extreme extra-pair mating system. BMC Ecology 9:15.

Kingma SA, Hall ML, Arriero E, Peters A (2010) Multiple benefits of cooperative breeding in purple-crowned fairy-wrens: a consequence of fidelity? J Anim Ecol 79:757-768.

Kingma SA, Hall ML, Peters A (2011a) Multiple benefits drive helping behaviour in a cooperatively breeding bird: an integrated analysis. Am Nat 177:486-495.

Kingma SA, Hall ML, Peters A (2011b) No evidence for offspring sex-ratio adjustment to social or environmental conditions in cooperatively breeding purple-crowned fairy-wrens. Behav Ecol Sociobiol 65:1203-1213.

Komdeur J, Kraaijeveld-Smit F, Kraaijeveld K, Edelaar P (1999) Explicit experimental evidence for the role of mate guarding in minimizing loss of paternity in the Seychelles warbler. Proc R Soc Lond B 266:2075-2081.

Lake PE (1981) Male genital organs. In: King AS, McLelland J (eds) Form and function in birds, Vol. 2. Academic Press, New York, pp 1-61

Lombardo MP (2001) Individual and seasonal variation in external genitalia of male Tree Swallows. Auk 118:789-795. 
Low M, Castro I, Berggen $\AA$ (2005) Cloacal erection promotes vent apposition during forced copulation in the New Zealand stitchbird (hihi): implications for copulation efficiency in other species. Behav Ecol Sociobiol 58:247-255.

Mulder RA, Cockburn A (1993) Sperm competition and the reproductive anatomy of male superb fairy-wrens. Auk 110:588-593.

Mulder RA, Dunn PO, Cockburn A, Lazenby-Cohen KA, Howell MJ (1994) Helpers liberate female fairy-wrens from constraints on extra-pair mate choice. Proc R Soc Lond B 255:223-229.

Nakamura M (1990) Cloacal protuberance and copulatory behaviour of the Alpine Accentor (Prunella collaris). Auk 107:284-295.

Nakatsuru K, Kramer DL (1982) Is sperm cheap? Limited male fertility and female choice in the lemon tetra (Pisces, Characidae). Science 216:753-755.

470 Olsson M, Madeson T, Shine R (1997) Is sperm really so cheap? Costs of reproduction in male adders, Vipera berus. Proc R Soc Lond B 264:455-459.

Peters A, Astheimer LB, Cockburn A (2001) The annual testosterone profile in cooperatively breeding superb fairy-wrens, Malurus cyaneus, reflects their extreme infidelity. Behav Ecol Sociobiol 50:519-527.

475 Pinheiro J, Bates D, DebRoy S, Sarkar D, R Development Core Team (2011): Nlme: Linear and nonlinear mixed effects models. R package version 3.1-102.

Pitnick S, Markow T (1994) Male gametic strategies: sperm size, testes size, and the allocation of ejaculate among successive mates by the sperm-limited fly Drosophila pachea and its relatives. Am Nat 143:785-819.

480 Pizzari T, Cornwallis CK, Løvlie H, Jakobsson S, Birkhead TR (2003) Sophisticated sperm allocation in male fowl. Nature 426:70-74.

R Development Core Team (2011) R: A language and environment for statistical computing. R Foundation for Statistical Computing, Vienna. Url: http://www.R-project.org 
Reyer HU, Dittami JP, Hall MR (1986) Avian helpers at the nest: are they physiologically castrated? Ethology 71:216-228.

Rowe M, Pruett-Jones S (2006) Reproductive biology and sperm competition in Australian fairywrens. Avian Poultry Biol Rev 17:21-37.

Rowe M, Bakst MR, Pruett-Jones S (2008) Good vibrations? Structure and function of the cloacal tip of male Australian Maluridae. J Avian Biol 39:348-354.

490 Rowe M, Swaddle JP, Pruett-Jones S, Webster MS (2010) Plumage coloration, ejaculate quality and reproductive phenotype in the red-backed fairy-wren. Anim Behav 79:1239-1246.

Rowley I, Russell E (1997) Fairy-wrens and Grasswrens. Oxford: Oxford University Press; 1997.

Rowley I, Russell E (1993) The purple-crowned fairy-wren Malurus coronatus. 2. Breeding biology, social organisation, demography and management. Emu 93:235-250.

495 Salt WR (1954) The structure of the cloacal protuberance of the Vesper Sparrow (Pooecetes gramineus) and certain other passerine birds. Auk 71:64-73.

Sax A, Hoi H (1998) Individual and temporal variation in cloacal protuberance size of male Bearded Tits (Panurus biarmicus). Auk 115:964-969.

Tuttle EM, Pruett-Jones S, Webster MS (1996) Cloacal protuberances and extreme sperm production in Australian Fairy-wrens. Proc R Soc Lond B 263: 1359-1364.

Webster MS, Tarvin KA, Tuttle EM, Pruett-Jones S. (2004) Reproductive promiscuity in the splendid fairy-wren: effect of group size and auxiliary reproduction. Behav Ecol 15:907915.

Wedell, N, Gage MJW, Parker G (2002) Sperm competition, male prudence and sperm-limited females. Trends Ecol Evol 17:313-320.

Wolfson A (1954) Notes on the cloacal protuberance. Seminal vesicle, and a possibly copulatory organ in male passerine birds. Bull Chic Acad Sci 10:1-23.

Wolfson A (1952) The cloacal protuberance - A means for determining the breeding condition in live male passerines. Bird Banding 23:159-165. 
Figure 1. CP size of dominant male purple-crowned fairy-wrens in relation to breeding stage. Breeding stages are categorised as non-breeding, pre-breeding (21 - 1 day before egglaying), incubation and dependent young. CPs of males captured during the non-breeding stage were significantly smaller than CPs of males in pre-breeding and incubation stage, but not

515 significantly different from CPs of males with nestlings and fledglings. Numbers denote sample sizes (number of observations) and error bars denote standard errors.

Figure 2. CP size of dominant male purple-crowned fairy-wrens in relation to the number of days from (before and after) the start of egg-laying by the within-pair female. (A) CPs of

520 dominant males are larger when the number of days from within-pair females initiating egglaying is shorter. (B) This effect is driven by an increase in CP size during the pre-breeding stage (21 - 1 day before egg-laying).

Figure 3. CP size of dominant male purple-crowned fairy-wrens in relation to extra-pair 525 mating opportunities. CPs of dominant males are larger, both in the (A) non-breeding stage and (B) pre-breeding stage, when more clutches were initiated along the same river ('population') in the month of capture of the focal male (extra-pair mating opportunities).

Figure 4. CP size of male purple-crowned fairy-wrens in relation to (A) mating 530 opportunities and (B) cuckoldry risk. (A) Subordinates with an unrelated dominant female (filled symbols) had on average a larger CP compared to subordinate males that lived with their mother (open symbols), but unrelated males were also older so that it is unclear whether this is an effect of within-group mating opportunities or the effect of age. (B) The reproductive competition unrelated helpers represent to the dominant male is not reflected in the $\mathrm{CP}$ size of the latter: 535 dominant males that did and did not have a subordinate that was unrelated to the dominant female 
had similar CP sizes. Numbers denote sample sizes (number of observations; all males captured during pre-breeding) and error bars denote standard errors. 


\section{FIGURES}

$540 \quad$ Figure 1.

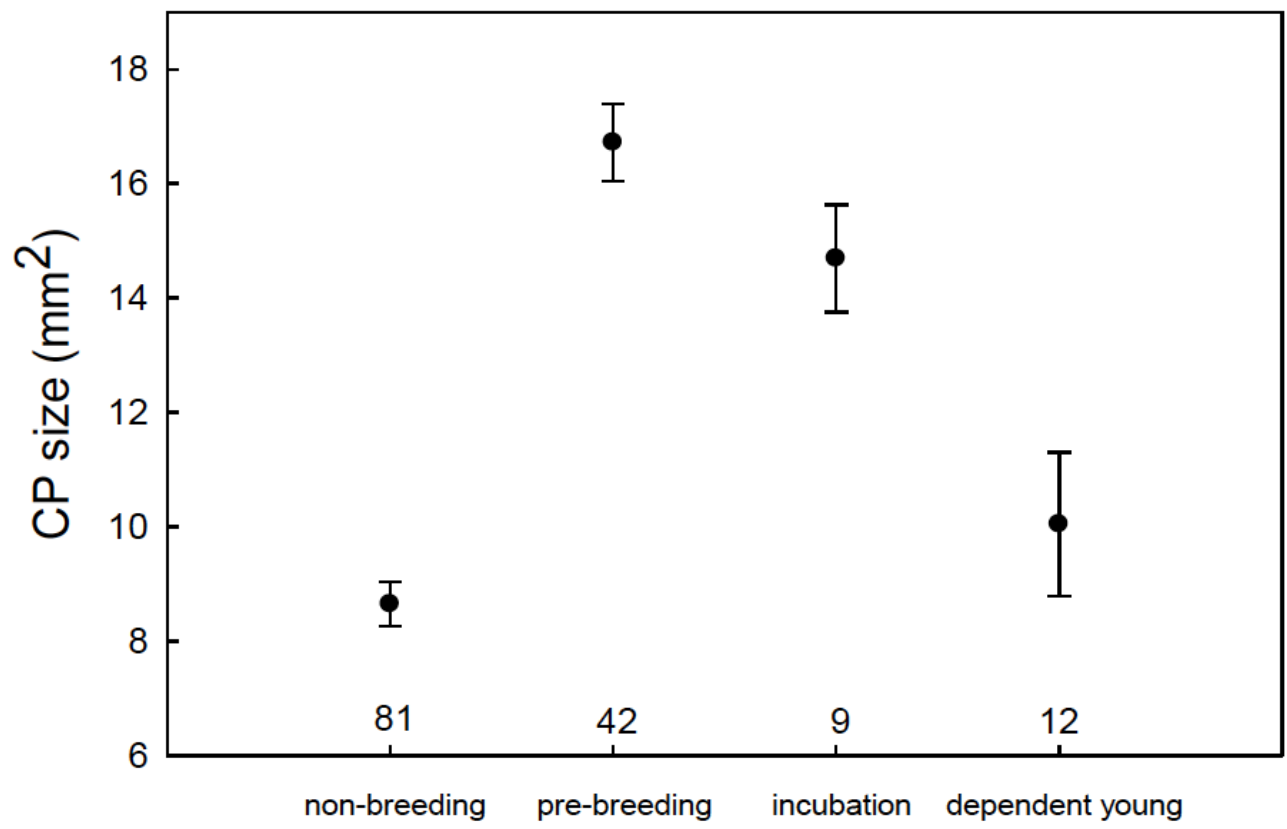


Figure 2.

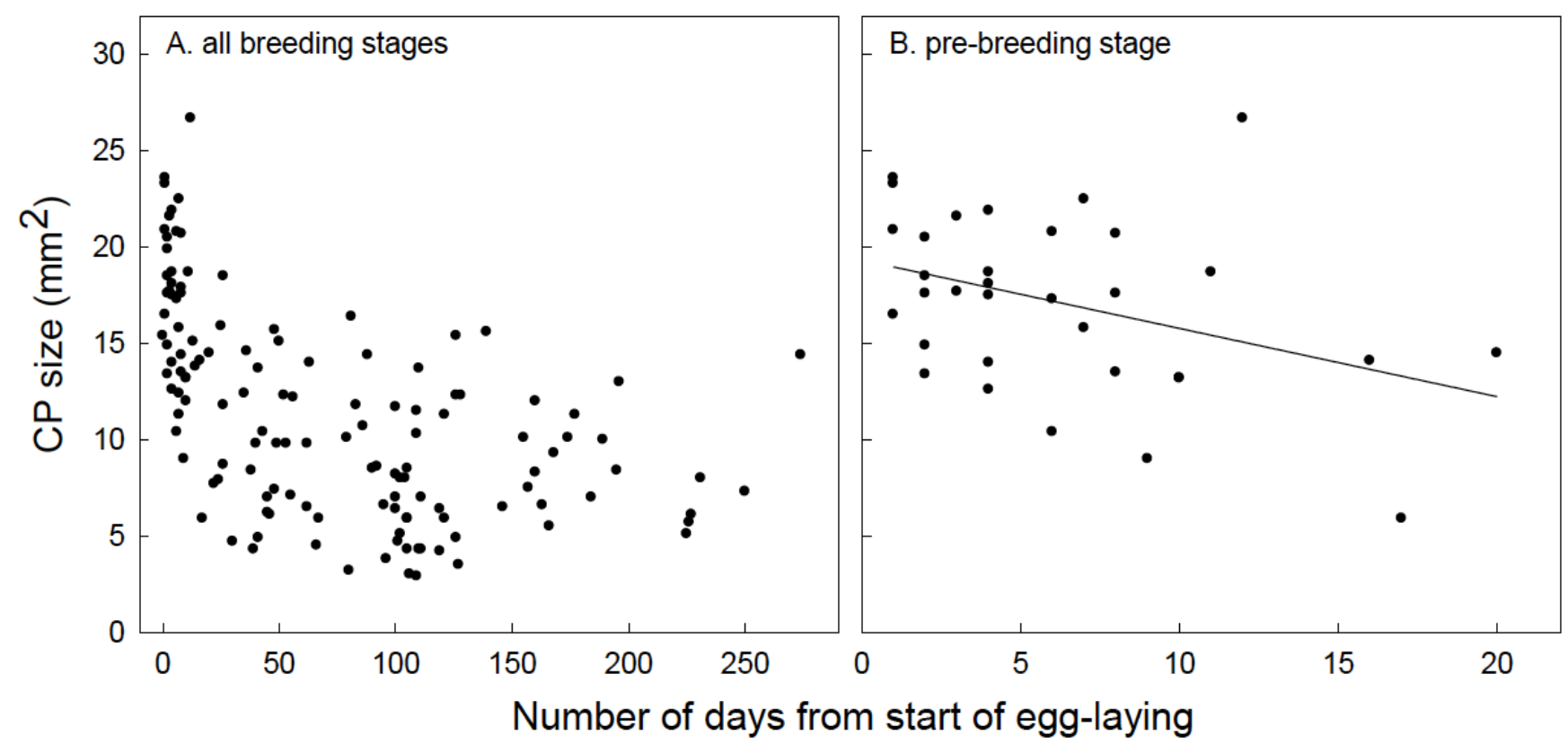


$545 \quad$ Figure 3.

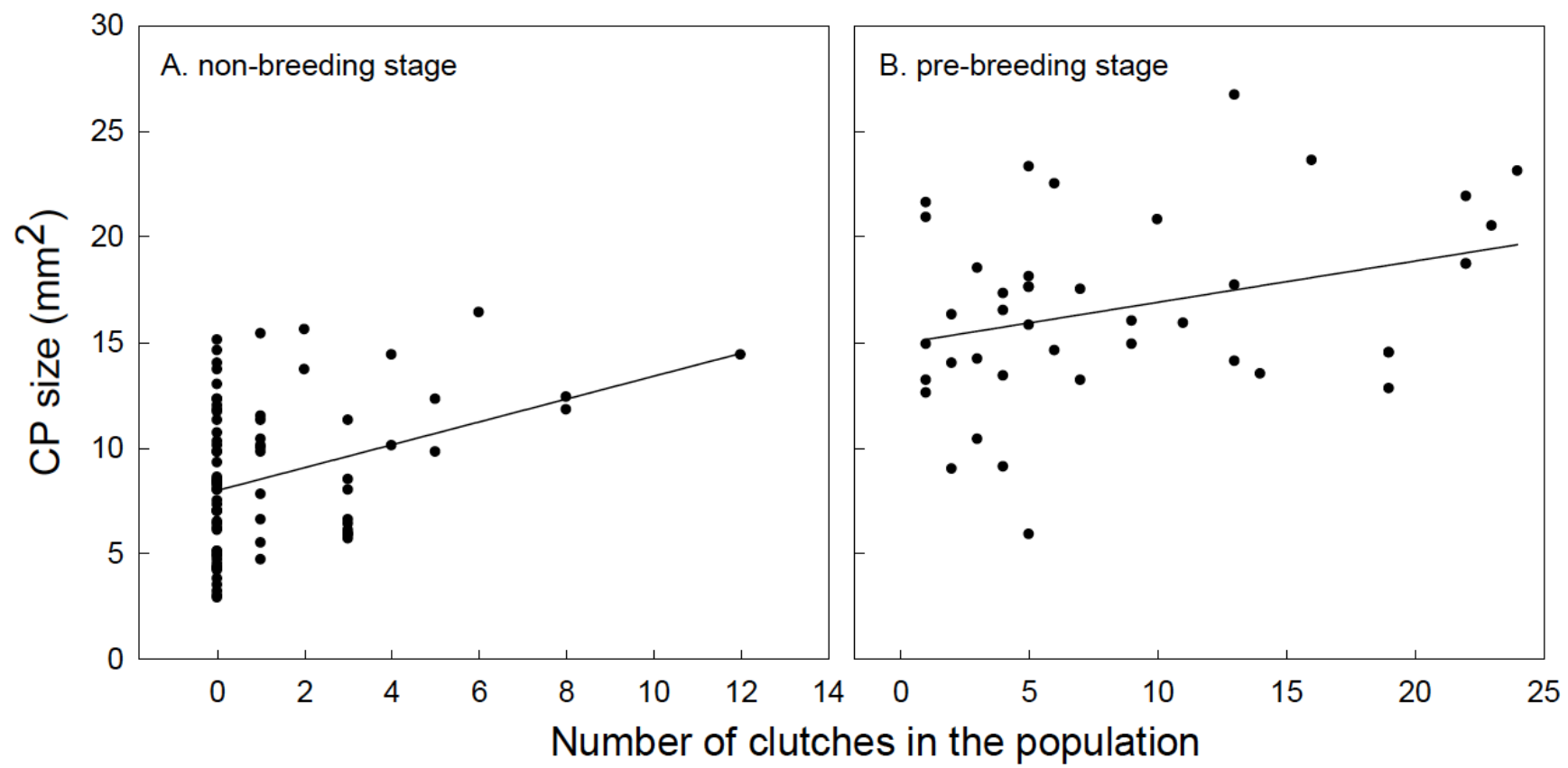


Figure 4.
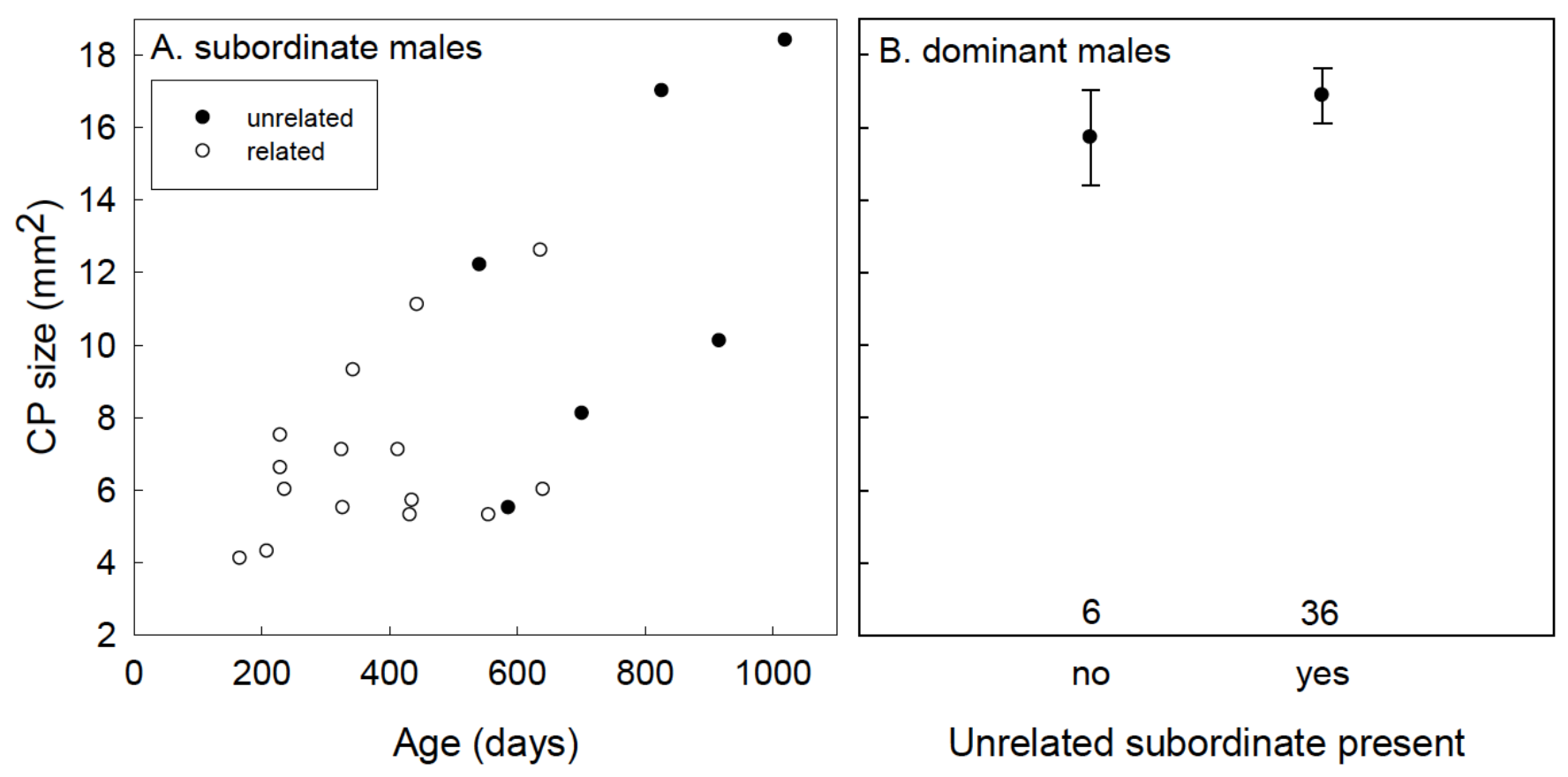

550 


\section{University Library}

\section{- M M N E R VA A gateway to Melbourne's research publications}

Minerva Access is the Institutional Repository of The University of Melbourne

Author/s:

Kingma, SA;Hall, ML;Peters, A

Title:

Sperm storage reflects within- and extra-pair mating opportunities in a cooperatively breeding bird

Date:

2012-08-01

Citation:

Kingma, S. A., Hall, M. L. \& Peters, A. (2012). Sperm storage reflects within- and extrapair mating opportunities in a cooperatively breeding bird. BEHAVIORAL ECOLOGY AND SOCIOBIOLOGY, 66 (8), pp.1115-1123. https://doi.org/10.1007/s00265-012-1363-y.

Persistent Link:

http://hdl.handle.net/11343/282691 